\title{
A Semantic Foundation for Role-Related Concepts in Enterprise Modelling*
}

\author{
João Paulo A. Almeida and Giancarlo Guizzardi \\ Federal University of Espírito Santo, Brazil \\ jpalmeida@ieee.org, guizzardi@loa-cnr.it
}

\begin{abstract}
In this paper, we provide a semantic foundation for role-related concepts in Enterprise Modelling. We use a conceptual modelling framework to provide a wellfounded underpinning for these concepts. We review a number of Enterprise Modelling approaches in light of the concepts described. This allows us to understand the various approaches, to contrast them and to identify problems in the definition and/or usage of these concepts.
\end{abstract}

Keywords: roles, actors, enterprise modelling, conceptual modelling, object modelling.

\section{Introduction}

The concept of "role" is present in several Enterprise Modelling approaches [15, 20, 22, 25]. In most of these approaches, enterprise activities are performed by entities which are called "actors", "agents" or "objects" and that can be said to play "roles" in these activities. Typically, the concept of role is used to define the responsibilities and properties that apply to "actors" while playing "roles" and what actions (or kinds of actions) are performed by which "actors".

"Roles" are also highly relevant when discussing the actions that are performed by users in interaction with a service or system and the service behaviour with respect to user interaction. In this case, it becomes necessary to define the (kinds of) actions that may be performed by particular (kinds of) users as well as the representation of users' identities and their properties in the scope of the service or system.

As discussed in [16] the role concept is based on a theatrical metaphor: "The text of a play is expressed in terms of lines and actions associated with various roles, which are declared initially in a cast-list. Putting the play on involves assigning actors to the various roles, although one actor may play several minor roles, and the actor playing a role may change during the run of the production. Identifying the roles rather than the actors obviously makes the script more reusable." Similarly, defining an enterprise or service model in terms of "roles", allows the model to remain stable in the presence of dynamic changes in role playing.
Although the term "role" is significantly present in Enterprise Modelling approaches, under close inspection, we can conclude that it often denotes different underlying concepts with different basic properties. Given the importance of roles in Enterprise Modelling, a clear semantic account for roles and role-related concepts is necessary and would serve as a basis for communication, consensus and alignment of the various approaches.

In this paper, we provide a semantic foundation for the role-related concepts for Enterprise Architecture and Enterprise Modelling language. Our claim is that some theories of conceptual modelling (as consolidated in [8]) provide a well-founded underpinning for these concepts, and allow us to harmonize competing proposals for them.

We review a number of Enterprise Modelling approaches in light of the semantic described, namely, Archimate, DoDAF, ARIS, BPMN and the RM-ODP (Enterprise Viewpoint). This allows us to contrast the approaches and to identify problems in the definition and/or usage of role-related concepts.

\section{Features of the role concept}

Steimann $[23,24]$ has identified a number of features for roles that appear throughout the object-oriented and conceptual modelling literature (e.g., $[4,5,6,7,8,17,18$, $28]$ ). We list each of those and introduce some examples to provide an intuitive notion of the role concept, prior to its rigorous definition:

1. "A role comes with its own properties and behaviour." For example, when John is enrolled as a student at the University of Twente, he has a grade point average (GPA), he can register to courses, receive grades, produce assignments, take exams, etc. This feature seems to suggest that roles can be regarded as a "type" characterizing a number of instances.

2. "Roles depend on relationships." For example, the roles of husband and wife as well as customer and supplier depend on the existence of a marriage or a business relationship. This is confirmed by the usage of the concept of role in the conceptual modelling literature as discussed in $[8,24]$ and as quoted in [5]: "as suggested by the work of Sowa and Guarino, a role is meaningful only in the context of a relation-

\footnotetext{
* This work is partly supported by FAPES (Fundação de Apoio à Ciência e Tecnologia do Espírito Santo) in the context of the INFRA-MODELA project, and partly supported by FACITEC (Fundo de Apoio à Ciência e Tecnologia do Município de Vitória) in the scope of the MODELA project.
} 
ship." This feature makes the concept of role distinct from that of a phase or a state [24].

3. "An object may play different roles simultaneously." For example, John can be a student and a husband at the same time.

4. "An object may play the same role several times, simultaneously." John can be a student at the University of Twente and at the Tai Chi Institute simultaneously.

5. "An object may acquire and abandon roles dynamically." John is still a Person after he graduates from the University of Twente.

6. "The sequence in which roles may be acquired and relinquished can be subject to restrictions." For example, John can only register in a graduate school after he has completed an undergraduate course.

7. "Objects of unrelated types can play the same role." For example, both a person (John) and an organization (the University of Twente) can play the role of customer in different business relationships.

8. "Roles can play roles." For example, John can play the role of teaching assistant for a particular course only if he is a student at the University of Twente.

9. "A role can be transferred from one object to another." For example, the commitments and responsibilities of the role of president are transferred from the incumbent president to his/her successor.

10. "The state of an object can be role-specific." If John is a student at the University of Twente and at the Tai Chi Institute simultaneously, John has a GPA for each of those relations.

11. "Features of an object can be role-specific." John attends all classes at this undergraduate course at the University of Twente but at the same time misses several classes in a row at the Tai Chi Institute.

12. "Roles restrict access." We consider this an implementation-oriented feature, considered by Steimann since he has surveyed object-oriented approaches in general. Since we are concerned with conceptual models for enterprise architectures, we do not include this feature further in our discussions.

13. "Different roles may share structure and behaviour." For example, both graduate students and undergraduate students have a student number, may register to courses, etc.

Features 14 and 15 contradict each other, showing that there is lack of agreement with respect to these features in the literature surveyed by Steimann:

14. "An object and its roles share identity."

15. "An object and its roles have different identities."

These features lead to the question of whether "John",

"John as a student of the University of Twente", "John as a student of the Tai Chi Institute" and "John as a husband" are the same, or whether there should be different identities for each of the roles John plays.

\section{Role-related concepts in conceptual modelling}

We proceed to identify a rigorous definition of the role concept, which requires some preliminary definitions. We use an extract from a philosophically and cognitively wellfounded reference ontology (foundational ontology) that has been developed in $[8,9]$.

First, we distinguish between conceptual entities called universals and individuals [8]. The notion of universal underlies the most basic and widespread constructs in conceptual modelling. Universals are predicative terms that can possibly be applied to a multitude of individuals, capturing the general aspects of such individuals. Individuals are entities that exist possessing a unique identity.

Figure 1 shows an extract of the foundational ontology adopted here (all generalization relations depicted in this figure are disjoint, forming a simple "tree-like" taxonomic structure for the entities considered in this model.)

This taxonomic structure reveals that an individual can be categorized as substantial or moment [10]. A moment is an individual that existentially depends on another individual, named its bearer. In the conceptual modelling literature, a moment is said to inhere in its bearer. For example, the symptoms of a patient are said to inhere in the patient, who bears the symptoms. In contrast, a substantial is an individual that does not inhere in other individuals, i.e., which is not a moment. Inherence is much stronger than a one-to-one relationship, since it implies existential dependence between individuals. We have that an individual $x$ is existentially dependent on another individual $y$ if, and only if, as a matter of necessity, $y$ must exist whenever $x$ exists. (A moment may also inhere in another moment, the moments forming a finite chain that ends with a substantial.)

In this paper, we characterize "actors", "agents" or "objects" as substantials and we explain the role-related notions in terms of moments. We use meta-properties of universals (namely, existential dependence, external dependence and rigidity) to clarify certain aspects of rolerelated concepts.

\subsection{Qua individuals and relators}

The taxonomic structure presented in Figure 1 reveals a kind of individual which is of particular importance to the definition of role (in gray on the right side of the figure): a "QuaIndividual".

An example discussed in [10] clarifies this concept. Suppose that John is married to Mary. John has a number of properties by virtue of being married to Mary. For 


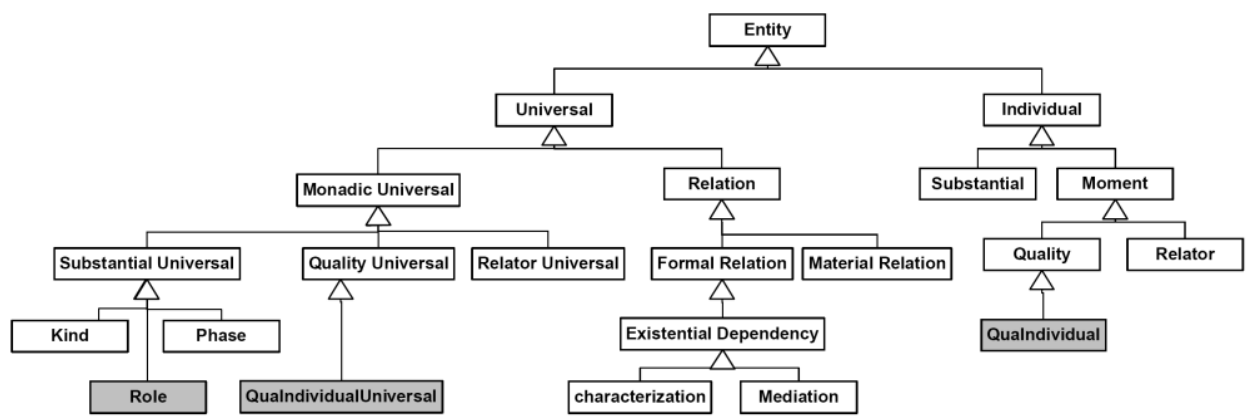

Figure 1 Extract of the foundational ontology adopted here from [10]

example, imagine all the legal responsibilities that John has in the context of this relation. These newly acquired properties are moments of John that inheres in him (and are hence existentially dependent on John). However, these moments also depends on the existence of Mary. This type of moment is called externally dependent moment. An externally dependent moment is an intrinsic moment (or quality) that inheres in a single individual but that is existentially dependent on (possibly a multitude of) other individuals external to its bearer (i.e., which is not the bearer's parts or intrinsic moments). In the example, this other individual is Mary.

In the case of an externally dependent moment $x$ there is always an event which is the foundation of $x$. Again, in the given example, we can think of a certain action $a l$ (the signing of a social contract) in which both John and Mary participate and which founds the existence of the externally dependent moments inhering in John. Now, we can define an individual that bears all externally dependent moments of John that share the same external dependencies and the same foundation. This individual is called a qua individual [17]. Qua individuals are, thus, a special type of complex externally dependent qualities. In this case, the complex quality inhering in John that bears all responsibilities that John acquires by virtue of the signing of a social contract can be named John-quahusband.

To continue with the same example, we can think about another qua individual Mary-qua-wife which is a complex moment bearing all responsibilities that Mary acquires by virtue of the same foundation and that albeit inhering in Mary are also existentially dependent on John. The qua individuals John-qua-husband and Mary-qua-wife are existentially dependent on each other. Now, we can define an aggregate composed of these two qua individuals that share the same foundation. This aggregate is called a relator.

\subsection{Role universals}

The taxonomic structure in Figure 1 also reveals a "Role" universal. A "Role" universal applies contingently to an individual that bears (at least one) qua individual of a certain type. In the example presented in the previous sub-section, we can say that John is not only an instance of a "Person" universal but also an instance of a "Husband" universal, while Mary is both an instance of a "Wife" universal. All instances of a "Husband" universal exhibit the behaviour required of a husband in a social contract (marriage).

At the same time John may play the role of student with respect to an "Educational Institution" for example, the University of Twente. In this case, John bears a qua individual John-qua-student, and is an instance of the "Student" universal (John can register to courses, receive grades, produce assignments, take exams, etc.). Further, John may also play the role of student with respect to other "Educational Institutions", for example, the Tai Chi Institute - bearing then qua individuals: John-qua-student of the University of Twente and John-qua-student' of the Tai Chi Institute.

We can say that roles universals can be restricted by certain allowed or admissible types, i.e., certain universals to which a role universal can apply. For example, in this case, we can say that the "Student" role can only be played by an instance of the kind "Person". A kind is the substantial universal which supplies a principle of identity for its instances and that is instantiated necessarily by its instances. Figure 2 shows a class diagram for this example, using the profile defined in [8]. The characterization association represents that instances of "PersonQuaStudent" inhere in an instance of "Student" (thus characterizing its behaviour).

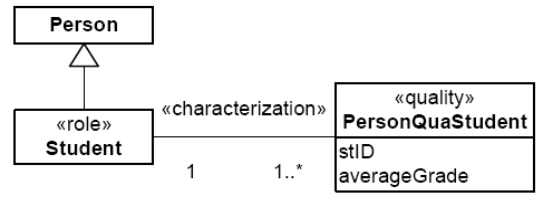

Figure 2 A role universal, its allowed type and a qua individual universal (from [8])

Figure 3 reveals the Enrolment relator universal (an instance of this universal includes an instance of 
"PersonQuaStudent"). The relator universal reveals that both an instance of "Student" and an instance of the "Education Institution" exhibit particular properties (shared behaviour) in the relation. Please note that properties are merely a dual way to represent behaviour.

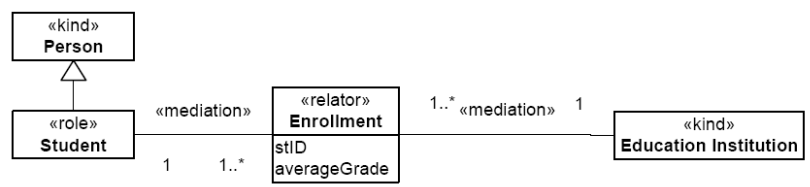

Figure 3 A role universal, its allowed type and a relator universal (from [8])

\subsection{Role mixin universals}

The conceptualization in [8] also allows for a notion of role mixin universal which captures commonalities in various role universals. This universal is used in a conceptual modelling design pattern for "roles with multiple disjoint allowed types" (see Figure 4). (We omit the description of role mixins from this paper, please see [8] for a comprehensive discussion and characterization of a role mixin as an anti-rigid non-sortal universal.) Intuitively, a role mixin universal allows us to add flexibility to a role universal, without tying its definition to a specific kind. In the example, it is possible to define a Customer independently of whether Persons or Organizations play that role.

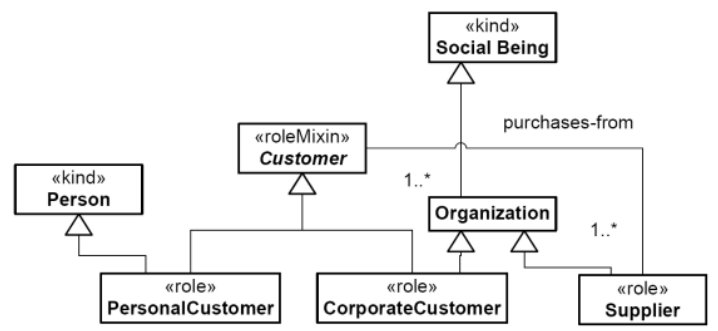

Figure 4 Modelling roles with multiple disjoint allowed types (an example from [8])

\subsection{Examples}

Table 1 summarizes the various examples presented throughout this paper and the concepts they illustrate.

\subsection{Role-related concepts in the foundations and the features presented by Steimann}

We can consider the foundations with respect to each of the features of role-related concepts as presented by Steimann:

1. "A role comes with its own properties and behaviour." Yes, a qua individual characterizes (with properties and behaviour) the substantials that play a particular role.

2. "Roles depend on relationships." Yes, a qua individual is externally dependent.

3. "An object may play different roles simultaneously." Yes, several qua individuals may characterize the same substantial.

4. "An object may play the same role several times, simultaneously." Yes, several qua individuals that characterize a substantial may be instances of the same universal.

5. "An object may acquire and abandon roles dynamically." Yes, a role universal applies contingently to substantials. In other words, a qua individual describes a complex of contingent properties of individuals.

6. "The sequence in which roles may be acquired and relinquished can be subject to restrictions." Yes, one can define conditions for the foundation of relators.

7. "Objects of unrelated types can play the same role." Yes, the mixin universal can be used in the design pattern for "roles with multiple disjoint allowed types".

8. "Roles can play roles." Yes, it is possible to restrict the admissible type of a role to another role.

9. "A role can be transferred from one object to another." Yes, this only requires one to define rules for the foundations of relators.

10. "The state of an object can be role-specific." Yes, see 1.

11. "Features of an object can be role-specific." Yes, see 1 .

13. "Different roles may share structure and behaviour." Yes, a role universal may specialize another role universal or role mixin universal.

With respect to contradicting 14 and 15 we can conclude:

14. "An object and its roles share identity." Yes, if one considers that roles are ultimately played by a substantial that carries a principle of identity.

15. "An object and its roles have different identities." Yes, the qua individuals have identities of their own.

\section{Role-related concepts in Enterprise Modelling}

In this section, we review role-related concepts in a number of enterprise modelling approaches (Archimate, DoDAF, ARIS, BPMN and RM-ODP). We contrast the definitions and usage of concepts in these approaches with the UFO-A conceptualization described in section 3 . 


\begin{tabular}{|c|c|}
\hline UFO-A & Example \\
\hline $\begin{array}{l}\text { role universal (a role universal applies(contingently) to } \\
\text { instances of the role's allowed type.) }\end{array}$ & Husband; Wife; Student; PersonalCustomer; CorporateCustomer. \\
\hline $\begin{array}{l}\text { role mixin universal (These universals apply (contingently) } \\
\text { to instances of disjoint admissible types.) }\end{array}$ & Customer \\
\hline $\begin{array}{l}\text { instance of the role universal (individual that bears a qua } \\
\text { individual) (instance of an admissible type for the roles } \\
\text { involved) }\end{array}$ & $\begin{array}{l}\text { John; } \\
\text { Mary; }\end{array}$ \\
\hline universals of the admissible types for particular roles & $\begin{array}{l}\text { Person (admissible type for roles Husband, Wife, Student, Personal } \\
\text { Customer); Organization (for CorporateCustomer); } \\
\text { Customer (for CorporateCustomer and PersonalCustomer); }\end{array}$ \\
\hline $\begin{array}{l}\text { qua individual (A qua individual is the instance that } \\
\text { characterizes the individual with certain behaviour in the } \\
\text { context of a relation to another individual.) }\end{array}$ & $\begin{array}{l}\text { John-qua-husband; Mary-qua-wife; John-qua-student (of the } \\
\text { University of Twente); John-qua-student' (of the Tai Chi Chuan } \\
\text { Institute). }\end{array}$ \\
\hline qua individual universal & Person-qua-Student; Person-qua-Husband; Person-qua-Wife. \\
\hline $\begin{array}{l}\text { the foundation of the qua individuals (and hence the } \\
\text { foundation of the relator, i.e. a founding action or behaviour.) }\end{array}$ & $\begin{array}{l}\text { the signing of the social contract; the act of enrolling at the university; } \\
\text { the act of enrolling at the Tai Chi Chuan Institute. }\end{array}$ \\
\hline relator (an aggregate of the qua individuals in the relation.) & $\begin{array}{l}\text { John and Mary's marriage; John's enrolment at the University of } \\
\text { Twente; John's enrolment at the Tai Chi Chuan Institute. }\end{array}$ \\
\hline relator universal & $\begin{array}{l}\text { Marriage (this kind of social contract); } \\
\text { Enrolment (this kind of social contract). }\end{array}$ \\
\hline individuals that are mediated by a relator & $\begin{array}{l}\text { John and Mary; John and the University of Twente; } \\
\text { John and the Tai Chi Chuan Institute. }\end{array}$ \\
\hline
\end{tabular}

\section{Table 1 Correspondence between role-related concepts in UFO-A and examples}

\subsection{Archimate}

In the Archimate Enterprise Architecture language [12, 15], the concepts of "business actor" and "business role" are introduced. A business actor is defined as an active entity that performs behaviour [15]. Examples of business actors include an individual person, a department and a business unit. A business role is identified with the purpose of making "the link between actors and behaviour more flexible." A business role is defined as that which "states which business behaviour is performed by a business actor that fulfils this role."

Intuitively, the definitions seem to imply that the business actor concept is a substantial and that the business role concept is a role universal that may be applied to actors (although the criteria of external dependency is not explicitly mentioned).

The language allows "actors" and "roles" to be related by what is called "assignment". Figure 5 shows an example of Archimate model with actors depicting "actors" and "roles". In this example, an "actor" named "Client" is assigned to the "role" named "Insurance buyer", which executes the behaviour "Buy insurance". Further, the actor "ArchiSurance" is assigned to the "role" named "Insurer", which executes the "Take out insurance" behaviour.

Although the definition for "actor" seems to imply that an "actor" is an individual, the language makes no distinction between the "actor" as an individual and a universal for "actors". This can be observed in the example show in Figure 5. The figure shows the "Archi-
Surance" "actor" which denotes a particular insurance company, i.e., it represents a particular substantial individual. Nevertheless, it also shows a "Client" "actor" which is certainly not tied to a particular client (such as "John") (otherwise the business process itself would be client-specific.) We can conclude that a "Client" in this case represents a universal for actual clients which may participate in the business process. Based on this example, we can state that the language lacks expressiveness with respect to the distinction between universals and individuals when considering the "actor" concept. Thus, this lack of expressiveness leads to a construct overload, which reduces the clarity of the language.

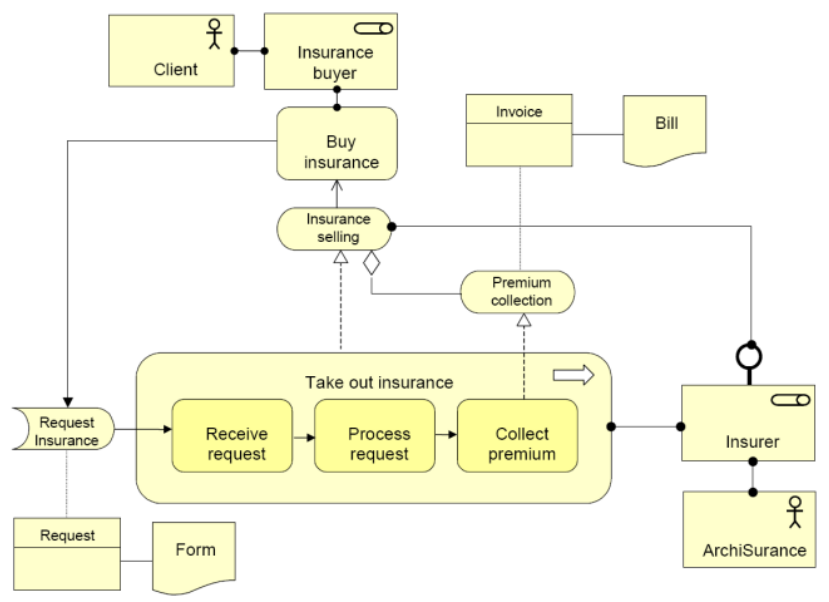

Figure 5 Archimate model (from [14]) 


\subsubsection{Concept Analysis: Interpretation A (Actors denote universals)}

A feasible interpretation to enable our analysis is to consider all "actors" in Archimate to denote universals, with certain "actors" representing universals that have only one instance (and, hence, are singletons, such as "ArchiSurance" in the example presented in Figure 5). Nevertheless, even with this interpretation, the language would not allow one to identify which universals are singletons and which are not.

Under this interpretation, we consider that the "assignment" relation represents a specialization/generalization relation between a role universal and its admissible type. In this particular example, instances of "Client" are the individuals that can play the role of "Insurance buyer", i.e., that can instantiate the "Insurance buyer" universal.

\subsubsection{Concept Analysis: Interpretation B (Actors denote individuals)}

An alternative interpretation would be to consider that the actor modelling element indeed represents individuals and that Figure 5 represents an abuse in notation and that in this case "Client" should be omitted from the model. This would be consistent with the usage of the "actor" modelling element in several examples in the Archimate documentation. See Figure 6 for an example of an Archimate model with a nesting of actors, all of which are individuals (nesting of actors in Archimate implies either aggregation or composition with no notational distinction possible).

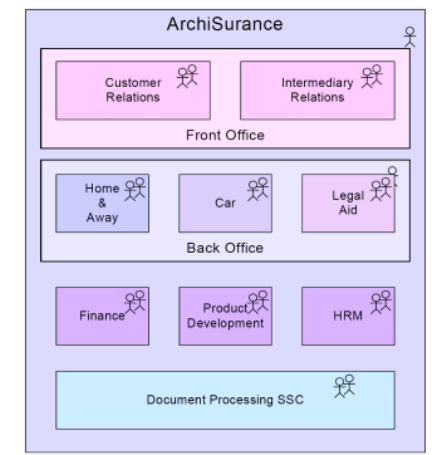

Figure 6 Organization model (from [12])

Another example that corroborates this interpretation is presented in Figure 7. The figure shows specific persons ("A. Smith", "D. Jones", and "M. Baker") as "actors" which are part of the departments of the insurance company.

Under interpretation $\mathrm{B}$, we consider the assignment relation (shown in Figure 7) to show that the actor is an instance of the role universal represented by the role modelling element. No statement is thus made about admissible types in general.

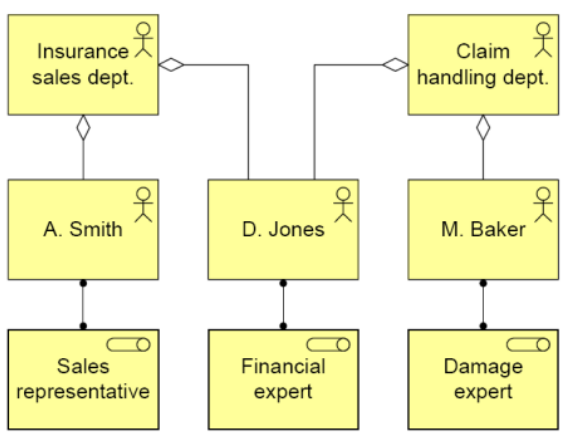

Figure 7 Organization model (from [12])

This interpretation would imply that Archimate cannot represent universals for actors (the resemblance of Figure 7 with the UML Class Diagram notation is unfortunate in this case, since the relations should be interpreted as links that relate the whole to the part).

\subsubsection{Generic relations}

The Archimate language has a number of generic relations which can be applied between a number of modelling elements. Nevertheless, the detailed semantics of these relations when applied to particular kinds of concepts is not always clarified. A particular example is the "specialization" relation.

The Archimate language reference manual [12] defines that "the specialization relationship can relate any instance of a [modelling] concept with another instance of the same concept." The case of specialization of roles is mentioned explicitly (e.g., 'junior' and 'senior' specializations of the same role [14]), thus corroborating our claim that roles are role universals. Nevertheless, the case of specialization of "actors" is not mentioned explicitly. Specialization of "actors" would be acceptable under interpretation A, but would be impossible under interpretation B (the specialization relation is a relation between universals).

Other relations are "aggregation" and "composition". These can be applied between "actors" (as shown in Figure 6 and Figure 7), between "roles", and also between "actors" and "roles" (as shown in Figure 8.) Please note that from this model it is impossible to derive the cardinality of the relation (i.e., should we interpret this model as stating that there can be multiple "Damage experts" in a "Claim handling department"?)

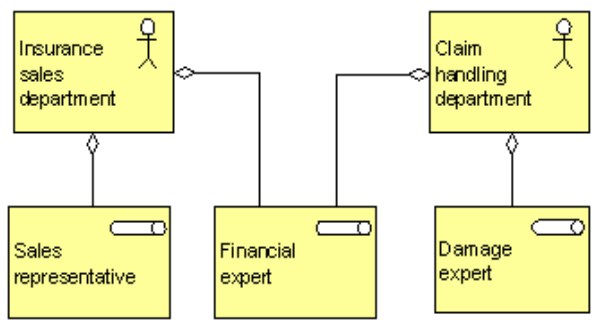

Figure 8 Relations between actors and roles [1] 
Although examples of organigrams such as the one presented in Figure 9 appear in the Archimate Resource Tree [1] and in examples of tools such as BizzDesign Architect [3], the semantics of the "relations" between "actors" is not discussed in the Archimate language reference manual. Ideally, these "relations" should be instances of material relations that are derived from relator universals. Relator universals would define the particular attributions of each of the relata, and their dynamics (creation and destruction) could be defined in the context of business processes.

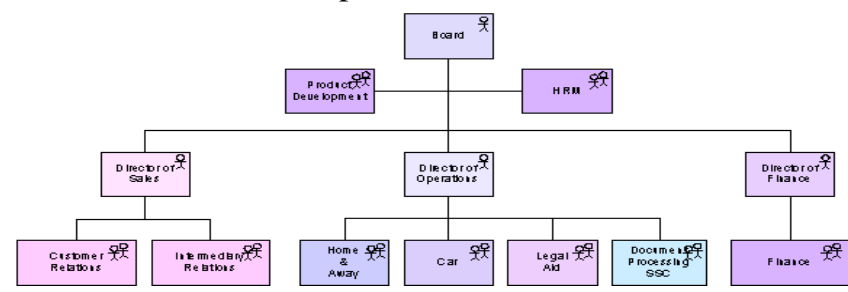

Figure 9 Example of Organigram [1]

\subsection{DoDAF}

The Department of Defense Architecture Framework (DoDAF) [25] defines two viewpoints that include rolerelated concepts. These are Operational Node Connectivity Description (OV-2) and Organizational Relationships Chart (OV-4).

In OV-2, "An operational node is an element of the operational architecture that produces, consumes, or processes information. What constitutes an operational node can vary among architectures, including, but not limited to, representing an operational/human role (e.g., Air Operations Commander), an organization (e.g., Office of the Secretary of Defense (OSD)) or organization type, i.e., a logical or functional grouping (e.g., Logistics Node, Intelligence Node), and so on. The operational node will also vary depending on the level of detail addressed by the architecture effort." [25]

In OV-4, "the Organizational Relationships Chart illustrates the command structure or relationships among human roles, organizations, or organization types that are the key players in an architecture." [25]

The following definitions are provided for OV-4: "Human Role - Skills are needed to perform the operational activities or business processes described in the architecture"; "Organization - An administrative entity with a, identity, structure, and mission."; "Organization Type - A Class of Organization"; "Organizational Relationship - relationships can include supervisory reporting, command and control relationships, and command-subordinate relationships."

Based on the definitions, we can, intuitively, interpret the "Human Role" concept as a role universal with an implicit admissible universal to represent humans. Further, we can interpret "Organization" as a substantial, and "Organization Type" as a kind. There is no concept for kinds or substantials when applied to model humans.

DoDAF proposes a number of UML styles for representing an architecture, including the aspects of the architecture that are related to roles and substantials. Figure 10 shows the proposed UML style for OV-2. Similarly to Archimate, "roles" are associated with the business processes in which they participate. Also, similarly to Archimate, the structuring of "roles" in "nodes" with the "aggregation" relationship suggests that nodes represent organizational units types in the context of which substantials that play the roles operate. (It is unclear from the documentation whether roles can be associated with multiple nodes directly.) We concluded that a "node" represents a kind and that a "role" represents a role universal. The representation in the lower part of Figure 10 confirms that by showing instances of "roles" and "nodes".

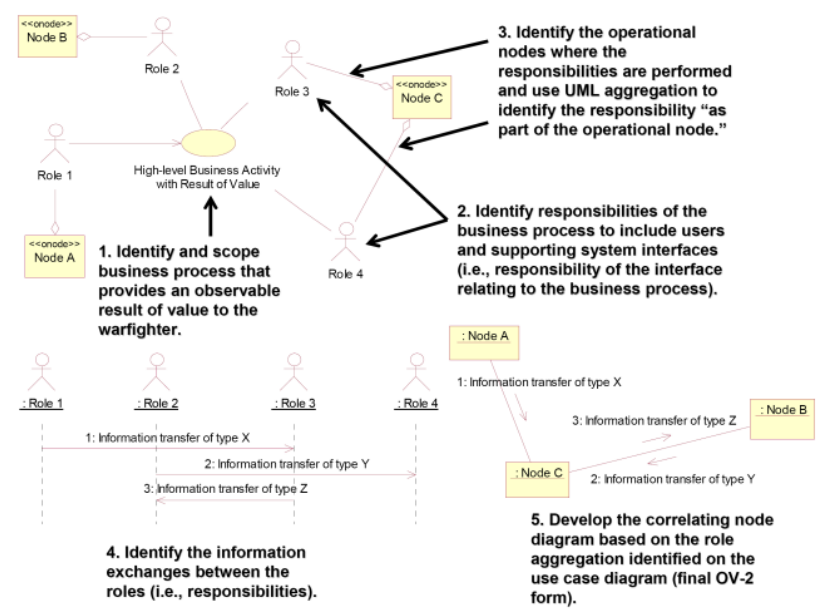

Figure 10 UML OV-2 template from [25]

Roles can be played by instances of other roles as can be seen in Figure 11. In this case, we interpret the relations depicted as representing that the "Mission Planner" "role" (a role universal) may apply to admissible type "Role 1".

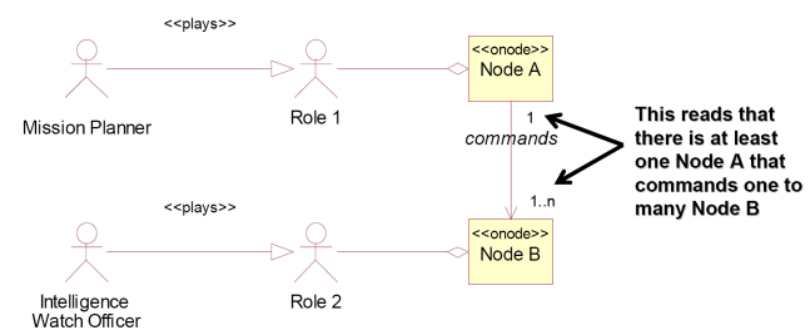

Figure 11 UML OV-4 Sample from [25]

The guidelines for UML usage in the DoDAF documentation are not prescriptive enough, and hence, a number of tools, represent DoDAF architecture in 
different styles. For example, MagicDraw provides a plugin for DoDAF using its own modelling elements. Figure 12 shows a screen shot of a model produced with this plug-in.

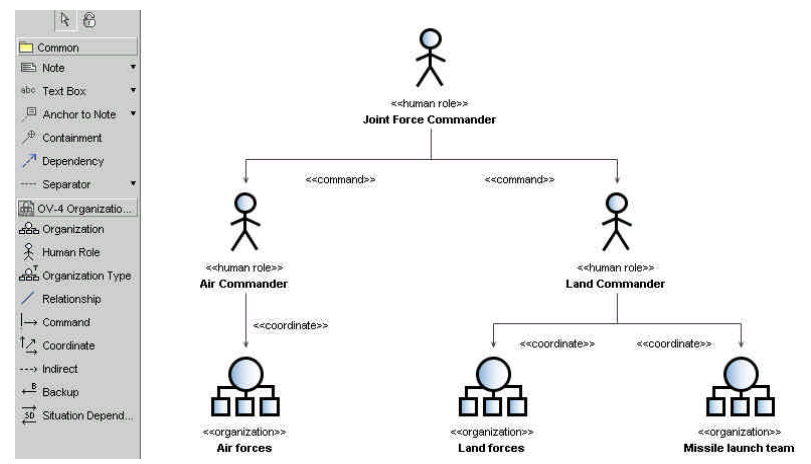

Figure 12 DoDAF OV-4 Organizational Relationships Chart in MagicDraw [19]

In the IBM Rational Approach to the DoDAF [27], there is no semantics associated with OV-4 diagrams. The document suggests the following with respect to an organizational structure chart: "Create a Freeform diagram and name it Organizational Structure. Add rectangles and label them for each organizational element to be represented. Use vertical relationships via solid lines to reflect command relationships, with higher authority at the top of the diagram. Show coordinating relationships using dashed lines."

The UML Profile for DoDAF/MODAF (UPDM) [21] defines an industry standard UML representation for DoDAF and MODAF compliant enterprise architectures. However, with respect to OV-4, the profile states that "this diagram represents information generally developed and maintained using techniques and tools better suited to the task than UML".

\subsection{ARIS}

The "Architecture of Integrated Information Systems" (ARIS) [22] framework is widely employed for the description of enterprise architectures.

ARIS includes the following role-related concepts: "Organizational Unit", "Organizational Unit Type", "Position", "Employee" and "Role".

The concept of "Organizational Unit" represents a substantial, instance of the "Organizational Unit Type", which we interpret as a kind. A "Position" is defined as the smallest organizational unit possible (a particular job position). If we interpret this definition literally, a "Position" represents an individual similarly to an organizational unit. "Positions" can be related to "Organizational Units" to represent responsibility (e.g., the CEO of IBM is "responsible for" the entire company) or to represent a whole-part relation.
An "Employee" is a particular individual (an instance of a universal that is not explicitly modelled.) A "Role" represents a role universal, all instances of which are necessarily "Employees", i.e., the only admissible type for "Roles" is the implicit universal that characterizes all "Employees".

The relation between "Roles" and "Positions" is rather indirect: when an "Employee" is related to a "Position" (the foundation for this relation is the hiring process), he/she plays the particular "Roles" that are somehow associated with the "Position".

Figure 13 shows an example of organigram in Aris, illustrating the usage of the concepts of "Organizational Unit", "Positions", "Employee" and "Role".

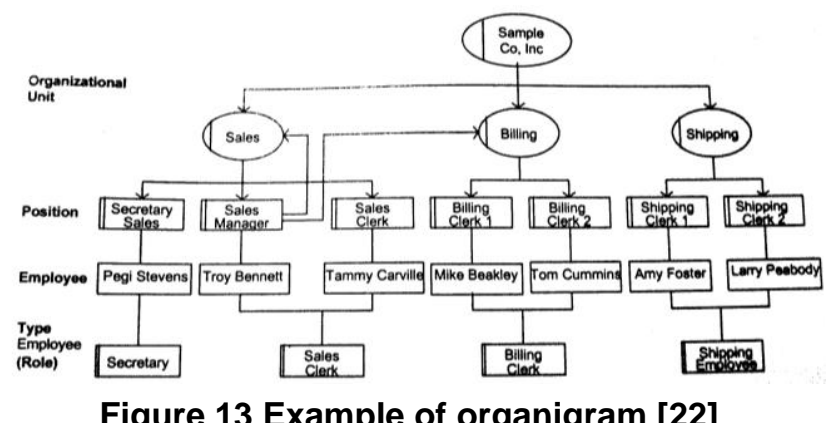

"Organizational units", "organizational unit types", "positions", "employees" and "roles" can be related to a business process or its activities through an "executed by" relation, as depicted in Figure 14.

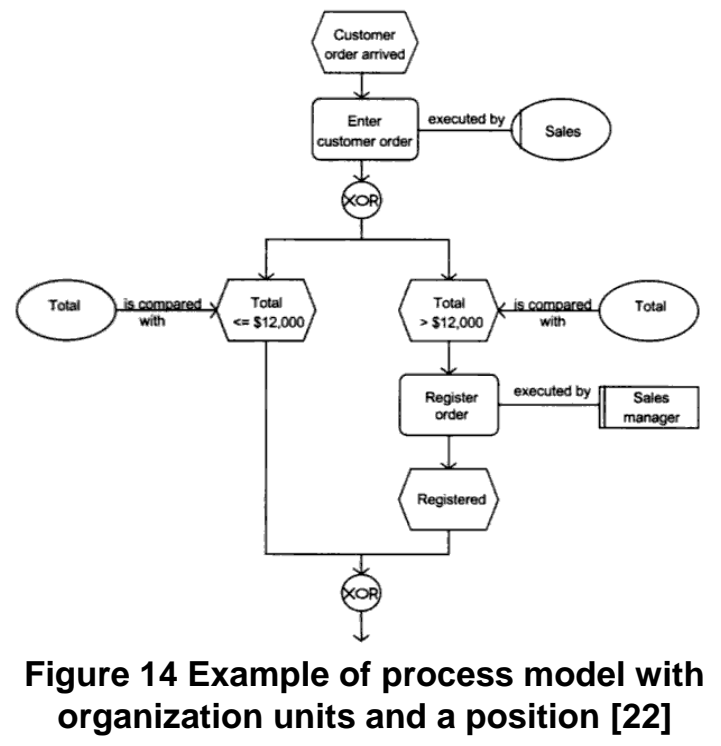

The semantics of these concepts and relations are not clearly documented in [22]. Thus, the analysis we have provided here must be considered as a first attempt to establish a consistent interpretation of the constructs based on usage examples. In future work, we intend to explore an interpretation of "Position" by using the concept of a 
normative description. In this alternative interpretation, a "Position" would characterize the behavior to which an "Employee" commits when assigned to a position. This would allow us to formally characterize not only the semantics of "Position" but also the semantics of the relations of "Positions" and other elements in the model. This alternative has not been explored here since the concept of normative description is not part of UFO-A, but rather of an extension of it, UFO-C [11].

\subsection{RM-ODP}

In our previous work [2], we have discussed the relation of the foundations presented here and the RM-ODP foundations. We have concluded that the RM-ODP provides a rich conceptualization when referring to the acts which constitute the foundation for roles. In the Enterprise Viewpoint is possible to describe "enterprise objects" as "communities" and detail their composition by using the concepts of "roles" and constituent "objects". We refer to [2] for further discussion on the topic.

\subsection{BPMN}

The Business Process Modelling Notation (BPMN) [20] focuses on business process modelling, and therefore does not provide constructs for organization modelling. Nevertheless, activities in a business process may be related by using the "Participant" model element to either an "Entity" or a "Role". Possible interpretations for these concepts are kind and role universal or role mixin universal.

\section{Additional Remarks}

\subsection{Roles versus kinds}

Consider the example where "John" studies at the "University of Twente". The "University of Twente" is an instance of the "Education Institution" universal. A definition of "Education Institution" is that it behaves in a certain way with respect to students (i.e., it executes some shared or collaborative behaviour with students.) It does so, however, necessarily, since the behaviour towards students is established in the definition of what an "Education Institution" is. The "University of Twente" is thus not playing the role of "Education Institution" in the context of a particular relation with a student. We believe there is no need to force the designer to use a role here or to imply that there is a role even if it is not modelled. (Of course one could always imagine that we could model the University of Twente as an "Institution" and then define an "Education Institution" role to be played by the university. Nevertheless, forcing this kind of construct would unnecessarily restrict the freedom of modellers.)

\subsection{Universals and individuals in models}

Consider now the presence of "singletons" in specifications, such as, e.g., the definition of roles such as "President of Brazil", which relates the object "Brazil" to a "Person" playing the role of "President". Again, one could define that there is a country B playing the role of "Brazil" or that there is an object "Brazil" playing the role of country, but this is beyond the point being made here namely that the modeller should have the freedom to define roles with respect to a particular object of special interest to the shared behaviour.)

\section{Conclusions}

We have contributed a semantic foundation for rolerelated concepts in Enterprise Modelling. Our contribution is well-positioned with respect to the literature in conceptual and object-oriented modelling ${ }^{2}$, thus possibly leading to a common foundation for these modelling domains.

We have found a number of difficulties in evaluating the selected enterprise modelling approaches, which reveals certain problems in the definition and potentially in the usage of some modelling elements in these approaches:

In the case of Archimate, the main difficulties refer to the interpretation of the concept of "actor". It was unclear from the documentation and from examples, whether the concept should be interpreted as a universal or an individual. We believe both universals and individuals for actors are relevant in enterprise modelling efforts (see section 5).

In the case of DoDAF, most issues relate to a lack of consensus on the language representation for the concepts, which restricts our analysis to the concepts as defined in the framework. We have concluded based on our analysis that there is no concept for kind or substantials when applied to model humans in DoDAF. This would make it impossible to model the interest in particular individuals (such as the allocation or deployment of persons to particular organizational units, as shown in Archimate and ARIS).

In the case of ARIS, both universals and individuals are provided for modelling organizational units. Individual human actors are also represented. The ARIS documentation has been hard to interpret (especially the role-related concepts as presented in [22]). Therefore, the

\footnotetext{
${ }^{2}$ For an extensive discussion on roles in the conceptual modelling literature that justify the UFO-A conceptualization see $[8,10]$. In $[8,10]$ the conceptualization provided here is defined formally, in order to allow for unambiguous interpretation of the intended semantics for concepts.
} 
semantics of the various modelling elements has been derived based on its usage in examples.

In none of the approaches, we could identify the distinction between the concepts of role universals and role mixin universals. In order to be able to model the design pattern for "roles with multiple disjoint allowed types" (which is one of the challenges presented in [24]), the approaches would have to collapse both concepts of role universals and role mixin universals in a single concept.

In all approaches, roles are used to represent the participation of actors in particular behaviours or processes, decoupling the definition of these behaviour or processes from particular instances of actors. None of the approaches, however, discuss the dynamics of role playing or provide modelling elements to describe how actors are assigned to roles dynamically (except the RM-ODP). The concept of qua-individual is very important in this respect and necessary to enable features $1,9,10$ and 11 of the list proposed by Steimann, i.e., those related to the properties and behaviour that individuals carry when playing a certain role. Qua individuals are also necessary to clarify the issue of identity and to solve the so-called "counting problem" [10].

Further work is needed to discuss the whole part relations for role-related concepts in details. Some discussions on this topic can be found in [8]. Further, some work is needed to relate the concepts discussed here to social concepts which are also available in enterprise modelling approaches such as commitments, contracts, goals, etc. [11]

\section{References}

1. Archimate Consortium, Archimate Resource Tree, available at http://www.telin.nl/NetworkedBusiness/Archimate/ART

2. J.P.A. Almeida, G. Guizzardi, "On the Foundation for Roles in RM-ODP: Contributions from Conceptual Modelling", $4^{\text {th }}$ WODPEC at EDOC 2007, IEEE CS Press, 2007.

3. BiZZDesigner, BiZZDesign Architect, available at http://www.bizzdesign.nl/html/bizzdesignarchitect_en.html

4. G. Genilloud, A. Wegmann, "A Foundation for the Concept of Role in Object Modelling", Proc. Int'l EDOC Conf. 2004, IEEE Computer Society Press, 2004.

5. G. Genilloud, A. Wegmann, "A New Definition for the Concept of Role, and Why it Makes Sense", OOPSLA Workshop on Behavioural Semantics, Oct. 2000.

6. N. Guarino, C. Welty, "Evaluating ontological decisions with OntoClean". Communications of the ACM, 45, 2, Feb. 2002, pp 61-65.

7. N. Guarino, C. Welty, "Identity and Subsumption. In The Semantics of Relationships: An Interdisciplinary Perspective”, eds. R. Green, C. Bean and S. Myaeng,. Amsterdam: Kluwer Academic, 2002, pp. 111-126.
8. G. Guizzardi, Ontological Foundations for Structural Conceptual Models, PhD Thesis, University of Twente, The Netherlands. TI-FRS No. 15, 2005.

9. G. Guizzardi, G. Wagner, "Towards Ontological Foundations for Agent Modeling Concepts using UFO", Lecture Notes on Artificial Intelligence (LNAI) 3508, SpringerVerlag, 2005.

10. G. Guizzardi, "Agent Roles, Qua Individuals and The Counting Problem", Software Engineering of Multi-Agent Systems, vol. IV, P. Giorgini, A.Garcia, C. Lucena, R. Choren (eds.), Springer-Verlag, 2006.

11. R.S.S. Guizzardi, G. Guizzardi, A. Perini, J. Mylopoulos, "Towards an Ontological Account of Agent Oriented Goals", Software Engineering for Multi-Agent Systems, Vol. V, Springer-Verlag, Berlin, 2007.

12. H. Jonkers et al., Architecture Language Reference Manual, Enschede: Telematica Instituut, 2003-2005.

13. ISO / ITU-T, Open Distributed Processing - Reference Model - Part 2: Foundations, International Standard ISO/IEC 10746-2, ITU-T Recommendation X.902, 1995.

14. H. Jonkers, (ed.), F. de Boer, M. Bonsangue, et al. A Concepts for Architectural Description TI/RS/2003/007, (ArchiMate/D2.2.1/v4.0), Telematica Instituut, 2003-2004.

15. M. Lankhorst et al., Enterprise Architecture at Work Modelling, Communication and Analysis, Springer, 2005.

16. P.F. Linington and W.F. Frank, "Specification and Implementation in ODP", Proc. 1st Int'l Workshop on Open Distributed Processing: Enterprise, Computation, Knowledge, Engineering \& Realisation (ICEIS), 2001, pp. 69-80.

17. C. Masolo, G. Guizzardi, L. Vieu, E. Bottazzi, R. Ferrario, "Relational Roles and Qua Individuals", AAAI Fall Symposium on Roles, an Interdisciplinary Perspective, USA, 2005.

18. K. Mulligan, B. Smith, A Relational theory of the Act. Topoi (5/2), 115-30, 1986.

19. No Magic, Magic Draw, available at http://www.magicdraw.com

20. Object Management Group. Business Process Modeling Notation BPMN 1.0, dtc/06-02-01, Feb. 2006.

21. Object Management Group, UML Profile for the Department of Defense Architecture Framework (DoDAF) and the Ministry of Defence Architecture Framework (MODAF), dtc/2007-08-02, August 2007.

22. A.-W. Scheer, ARIS - Business Process Modeling, Third Edition, Springer, 1999.

23. F. Steimann, A Radical Revision of UML's Role Concept, in UML 2000: Proceedings of the 3rd International Conference, Springer-Verlag, 2000, pp. 194-209.

24. F. Steimann, "On the representation of roles in objectoriented and conceptual modelling," Data and Knowledge Engineering, vol. 35, pp. 83-106, 2000.

25. U.S. Department of Defense, DoD Architecture Framework Version 1.5, Volume II: Product Descriptions, April 2007.

26. A. Wegmann, G. Genilloud, "The Role of 'Roles' in Use Case Diagrams", UML 2000: Proceedings of the 3rd International Conference, Springer-Verlag, 2000.

27. C. Widney, An IBM Rational Approach to the Department of Defense Architecture Framework (DoDAF), January 2006.

28. R.J. Wieringa, W. de Jonge, P.A. Spruit. "Using dynamic classes and role classes to model object migration". Theory \& Practice of Object Systems, 1(1), 1995, pp 61-83. 